\title{
Femmes et sida : regards croisés en sciences sociales
}

\author{
Nathalie BAJOs et Geneviève PAICHELER
}

Plus de trois décennies après l'identification des premiers cas de sida, l'épidémie continue de progresser inexorablement et touche de plus en plus les femmes dans tous les pays du monde, même si cette féminisation semble stabilisée depuis peu en France. Féminisée, l'épidémie s'est également précarisée et concerne, notamment dans notre pays, les femmes immigrées d'Afrique sub-saharienne. De fait, et cela revient de façon lancinante dans ce numéro spécial, c'est bien la vulnérabilité, déclinée sous de multiples formes, qui constitue le terreau de l'extension de l'infection à VIH.

Les sciences sociales auraient-elles pu anticiper cette évolution, qui devient maintenant si saillante, au regard du mode de transmission très majoritairement sexuelle de l'infection? La réponse est positive, si l'on porte un regard rétrospectif à l'aune des connaissances scientifiques dont on dispose aujourd'hui. Mais au début de l'épidémie, tant les cadres d'interprétation que les outils méthodologiques ne permettaient pas d'apporter une attention suffisante à sa féminisation, qui progressait pourtant à bas bruit. Plusieurs logiques se conjuguent pour rendre compte du retard pris dans ce domaine. L'analyse de ces logiques permet d'en comprendre le sens et d'identifier les questions de recherches qui demeurent pendantes aujourd'hui.

Ce numéro spécial n'est, espérons-le, qu'un premier jalon pour aborder de front la question de la place des femmes dans l'épidémie de l'infection à VIH. Fondé sur les données concernant les femmes dans des recherches qui, pour la plupart d'entre elles, n'étaient pas centrées sur cette question, il permet d'entreprendre une première démarche synthétique, tout en gardant à l'esprit que cette question n'a été le plus souvent que liminaire dans les investigations.

II faut tout d'abord rappeler que les premiers cas de sida identifiés ont touché des hommes ayant des rapports homosexuels. Les catégories épidémiologiques se sont construites sur la base des caractéristiques de l'épidémie à ses débuts dans les pays occidentaux, à partir de cas majoritairement masculins, et sont toujours de mise aujourd'hui. Certes, il importe d'assurer la comparabilité des données dans le temps pour être à même de suivre l'épidémie, mais force est de constater que la typologie actuelle conduit à classer la très grande majorité des femmes séropositives dans la même catégorie de " femmes hétérosexuelles ", sans autre précision. Une catégorie aussi peu discriminante n'éclaire guère l'analyse de l'évolution de l'épidémie chez les femmes, pas plus qu'elle ne favorise la réalisation de recherches sur des sous-groupes particuliers de femmes. En outre, les typologies épidémiologiques sont basées sur une logique de facteurs de risque (transmissions homosexuelle, hétérosexuelle, transmission sanguine etc), ce qui exclut la possibilité de caractériser les femmes atteintes ne présentant pas les facteurs de risque identifiés, comme les femmes monopartenaires en couple stable ou les femmes ayant des rapports homosexuels exposées lors de rapports hétérosexuels, pourtant particulièrement touchées par les maladies sexuellement transmissibles. C'est la raison pour laquelle il est plus pertinent d'appréhender l'exposition au risque de transmission du VIH, pas uniquement en termes de facteurs de risque figés et toujours lacunaires, mais en considérant l'exercice de la sexualité dans son contexte.

Le contenu des campagnes de prévention n'est d'ailleurs pas sans lien avec cette catégorisation épidémiologique. Les femmes ont été considérées au départ comme des cibles secondaires, du fait du profil initial de l'épidémie. Ce n'est que très progressivement que la communication a traité de la spécificité des femmes dans la sexualité et dans la reproduction, abordant tardivement la question de la transmission mère-enfant. C'est également progressivement que la vulnérabilité des femmes a été reconnue et, surtout, que les femmes les plus exposées au risque sexuel, originaires des Antilles ou d'Afrique sub-saharienne, ont été la " cible " de communications. En revanche, les femmes ont été très tôt mises en avant lorsque la communication se centrait sur la solidarité envers les personnes atteintes, cette attitude renvoyant "naturellement " à un de leurs rôles traditionnels.

Indéniablement, les représentations sociales de la sexualité féminine ont fortement marqué les orientations de recherche et la prévention, les femmes étant perçues comme ayant soit une sexualité "dévoyée " qui en faisait des cibles de l'épidémie (comme "par définition " les prostituées - qui sont d'autant plus en danger de contamination qu'elles sont dans une situation précaire ou clandestine -, les usagères de drogues, qui échangent leur produit contre des relations sexuelles, ou encore les femmes migrantes censées avoir de nombreux partenaires), soit une sexualité conjugale, supposée à l'abri de tout risque. C'est au regard d'une sexualité perçue comme dangereuse ou non que les femmes ont été appréhendées et non du point de vue de leurs spécificités sociales.

C'est bien une conjonction de logiques de nature différente, contradictoires pour certaines, qui contribue à rendre compte du retard pris dans les recherches sur "Femmes et $\mathrm{VIH} /$ sida ". Pour autant, il serait faux de dire que rien n'a été entrepris dans ce domaine. Quelques recherches en sciences sociales financées par l'Anrs ont 
porté spécifiquement sur les femmes, en particulier les femmes prostituées, les femmes séropositives et, récemment seulement, les femmes immigrées. De nombreuses autres recherches ont permis de tirer des enseignements sur cette thématique, notamment les grandes enquêtes sur la sexualité, dont la plus récente analyse le contexte de la sexualité en France, celles sur les personnes vivant avec le $\mathrm{VIH} /$ sida (enquête Vespa), sur les usagères de drogues (Coquelicot), les enquêtes KABP France, Antilles-Guyane, migrants, et de nombreuses recherches qualitatives permettant de comprendre certains processus en finesse et de travailler sur des terrains difficiles.

II serait illusoire de vouloir lister de manière exhaustive tous les enseignements de ces recherches, mais on peut néanmoins les regrouper en trois catégories.

- Tout d'abord, il est clair aujourd'hui que c'est dans une perspective de "situations à risque ", bien plus que de " comportements à risque ", qu'il convient d'appréhender la question de la prise de risques en matière de sexualité. Et avec ce changement de paradigme, la problématique des rapports sociaux, de genre avant tout, mais aussi de classe, de " race ", d'âge, et de leur articulation, trouve ici toute sa pertinence. Ces situations à risque sont le reflet de la violence, tant symbolique que bien réelle, qui s'exerce à l'encontre de nombreuses femmes et qui contribue à renforcer leur vulnérabilité.

- Le contexte social reste marqué par des inégalités de sexe importantes dans différentes sphères (éducation, travail, famille, domestique, politique), qui construisent le rapport à la sexualité et à la prévention. Inégalités qui prévalent toujours dans le champ de la sexualité où, comme le montre l'enquête Contexte de la sexualité en France, même si les pratiques des femmes et des hommes se rapprochent, les représentations de la sexualité continuent de s'articuler autour d'un clivage pensé comme naturel entre une sexualité féminine affective et des besoins sexuels masculins.

- Enfin, il devient de plus en plus manifeste que, comme le soulignaient déjà les anthropologues au début des années 1930, ce qui se joue dans la sexualité est subordonné aux enjeux de la reproduction d'autres rapports sociaux, économiques et politiques.

Cette (triple) perspective invite à dénaturaliser la féminisation de l'épidémie et à appréhender aujourd'hui les enjeux de l'infection à VIH chez les femmes en termes de cumul de vulnérabilités, sociales, économiques et culturelles. Cumul de vulnérabilités qui structure aussi bien la sexualité et les pratiques préventives que les trajectoires de vie affectives, sexuelles, sociales et professionnelles des personnes atteintes. Cette perspective conduit ainsi à sortir d'une approche misérabiliste trop souvent de mise dans les discours sur les femmes, bien que contredite par les résultats des nombreuses recherches présentées dans ce numéro spécial.

D'une manière générale, les recherches montrent que l'infection à VIH accroît la vulnérabilité des femmes, tant au niveau des enjeux préventifs qu'à celui de leurs parcours de vie et de santé. L'absence de moyens de prévention relevant de la seule responsabilité des femmes, à l'exception du fémidon (préservatif féminin) qui reste peu diffusé et utilisé, n'est pas sans contribuer à cette vulnérabilité.
Les recherches sur les microbicides sont à ce jour encore problématiques. II s'agit pourtant d'un enjeu fondamental, d'autant que le recours à la circoncision masculine, qui devrait se développer, pourrait conduire certains hommes à relâcher leur démarche de prévention.

Lorsqu'elles sont infectées par le VIH, les femmes accèdent plus tardivement au système de santé et sont moins observantes au plan thérapeutique, car les effets secondaires des traitements sont plus marqués chez elles. II leur est également particulièrement difficile d'informer leur partenaire de leur statut sérologique, même si les gynécologues qui les conseillent disposent d'une expérience en matière d'information des partenaires en cas de maladies sexuellement transmissibles. Leur vie affective est plus problématique que celles des hommes quand elles sont délaissées par leur partenaire et elles affrontent la solitude ou font face au veuvage, si leur conjoint était également infecté. Enfin, elles exercent des activités professionnelles sous qualifiées. Un tel contexte de vie est associé à des phases de dépression plus fréquentes et le soutien des associations apparaît particulièrement salutaire.

Cependant, il existe des exceptions à ce noir tableau : tous les couples ne se défont pas et l'espoir renaît du fait de l'efficacité des traitements et permet à celles qui le souhaitent d'avoir des projets de maternité qui ne rencontrent plus la réticence des médecins, comme avant l'arrivée des thérapies antirétrovirales, il y a quelque dix ans.

Les recherches consacrées aux migrantes offrent un prisme supplémentaire d'analyse. Elles montrent que la situation de plus grande précarité économique, sociale, voire affective les expose fortement à la transmission du VIH, d'autant qu'elles évoluent dans des réseaux de socialisation où la prévalence de l'infection est particulièrement élevée. Une fois atteintes, elles rencontrent plus de difficultés à réaliser leur projet de maternité, mais également pour se soigner. Cette situation de domination sociale, peu favorable à une prise en charge du risque et de la maladie, se retrouve également chez les femmes des départements français d'Amérique, ou dans un territoire autrefois colonisé comme la Nouvelle-Calédonie. Mais ces femmes parviennent aussi à utiliser au mieux les ressources dont elles disposent, comme le font par exemple certaines femmes sans domicile, en situation de très grande précarité.

Ce numéro spécial, coordonné par Geneviève Paicheler et Nadine Job-Spira, propose des regards croisés sur divers enjeux, différents groupes de femmes et plusieurs formes de vulnérabilités, regards qui permettent de traiter cette question centrale du cumul des vulnérabilités, sur la base des recherches financées par l'Anrs et en consacrant, exception qui confirme la règle, une contribution à des actions de terrain.

Soulignons enfin que l'implication de l'Anrs sur la question « Femmes et VIH » ne se limite pas aux sciences sociales et concerne bien évidemment les enjeux épidémiologiques, cliniques et fondamentaux de cette infection. La collaboration entre chercheurs de différentes disciplines a permis d'enrichir les approches respectives et de mieux comprendre ainsi les enjeux auxquels nous devons faire face. Elle se poursuivra dans le futur. 